Discrete Comput Geom 38:573-594 (2007)

DOI: $10.1007 / \mathrm{s} 00454-007-1354-3$

\title{
How to Exhibit Toroidal Maps in Space*
}

\author{
Dan Archdeacon, ${ }^{1}$ C. Paul Bonnington, ${ }^{2}$ and Joanna A. Ellis-Monaghan ${ }^{3}$ \\ ${ }^{1}$ Department of Mathematics and Statistics, University of Vermont, \\ Burlington, VT 05405, USA \\ dan.archdeacon@uvm.edu \\ ${ }^{2}$ Department of Mathematics, University of Auckland, \\ Auckland, New Zealand \\ p.bonnington@auckland.ac.nz \\ ${ }^{3}$ Department of Mathematics, Saint Michael's College, \\ Colchester, VT 05439, USA \\ jellis-monaghan@smcvt.edu
}

\begin{abstract}
Steinitz's theorem states that a graph is the 1-skeleton of a convex polyhedron if and only if it is 3-connected and planar. The polyhedron is called a geometric realization of the embedded graph. Its faces are bounded by convex polygons whose points are coplanar.

A map on the torus does not necessarily have such a geometric realization. In this paper we relax the condition that faces are the convex hull of coplanar points. We require instead that the convex hull of the points on a face can be projected onto a plane so that the boundary of the convex hull of the projected points is the image of the boundary of the face. We also require that the interiors of the convex hulls of different faces do not intersect. Call this an exhibition of the map. A map is polyhedral if the intersection of any two closed faces is simply connected. Our main result is that every polyhedral toroidal map can be exhibited. As a corollary, every toroidal triangulation has a geometric realization.
\end{abstract}

\section{Introduction}

Steinitz's theorem [29] characterizes those graphs that are 1-skeletons of convex polyhedra in 3 -space $\left(\mathbb{R}^{3}\right)$ : they are the 3 -connected planar graphs. We call the polyhedron a geometric realization of the graph (precise definitions of these and other terms are given in Section 2). Loosely speaking, the vertices of the graph are represented by points in

\footnotetext{
* The authors acknowledge financial support from the Marsden Fund administered by the Royal Society of New Zealand and the New Zealand Institute of Mathematics and its Applications.
} 
Euclidean 3-space, the edges are straight-line segments joining these points, all points in a face are coplanar, the faces are convex, and distinct faces intersect only along their common boundaries. Geometric realizations are used in rendering computer graphics, where three-dimensional structures are represented by points in space with convex faces.

Suppose that we are given a graph embedded on a surface of higher genus. When does this embedding have a geometric realization? The problem, restricted to triangulations, was first proposed by Grünbaum [17, Exercise 13.2.3], who conjectured that "Every closed orientable triangulated 2-manifold without boundary can be embedded geometrically in three-dimensional Euclidean space $\mathbb{R}^{3}$ " (see also [9]). This conjecture was recently disproven by Bokowski and Guedes de Oliveira [6], who showed that a certain triangulation of the complete graph $K_{12}$ on a surface of genus 6 cannot be realized geometrically. Brehm and Schild [8] showed that every triangulation of the torus does have a realization in $\mathbb{R}^{4}$. We refer the reader to [4], [18], [21], and [22] for further results on the realizability of certain manifolds.

In this paper we focus on graphs embedded on the torus, also called "maps" on the torus. An interesting example is the geometric realization in $\mathbb{R}^{3}$ of the complete graph $K_{7}$ (uniquely) embedded on the torus. This is commonly attributed to Császár [11], who discovered it independently, and is known as Császár's Polyhedron. Reay notes it was also known to Möbius. For a popular account and details on how to build a model, see [14] and [32]. Bokowski and Eggert [5] found all realizations of this polyhedron.

One of the results in this paper, Corollary 1.2, proves Grünbaum's conjecture for triangulations of the torus. This follows up on work of Altshuler [1], who found geometric embeddings of a large class of toroidal triangulations; namely, those that had a special type of Hamiltonian cycle. Similarly, Lavrenchenko (personal communication) also found geometric realizations of those toroidal triangulations where deleting two vertices gives a set of triangles that can be realized in the plane.

Some work has also been done on geometric realizations of toroidal maps that are not triangulations. For example consider the Heawood map, which is the dual of the embedding of $K_{7}$ on the torus. Figure 1 shows this map; here and in subsequent figures with dashed borders the top side is to be identified with the bottom side and the left side with the right so as to form the torus. (The role of the dashed and heavy edges in this figure will be explained in Section 7.) It has 14 vertices, each of degree 3, and each face

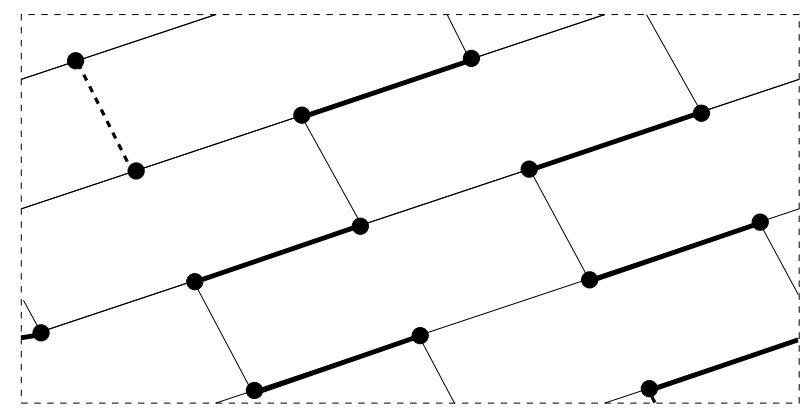

Fig. 1. The Heawood map. 
is bounded by a hexagon. Can we realize this map in 3-space such that for each face all of the interior points are coplanar, and the interiors of the faces do not intersect? The answer is yes, as found by Szilassi ([30], as reported in [15] and [33]).

The faces of Szilassi's polyhedron are non-convex. For completeness, we outline a proof that if the vertices of each face are coplanar and the faces have disjoint interiors, then some face must be non-convex (see also [3], [25], and Exercise 11.1.8(2) in [17]). Suppose that we had such a representation by convex faces. Let $f_{1}$ be one of the faces, and consider a representation in $\mathbb{R}^{3}$ with $f_{1}$ in the $x y$-plane. Any other face $f_{j}$, adjacent to $f_{1}$, lies entirely in the upper half-space with $z \geq 0$, or entirely in the lower half-space with $z \leq 0$. Since any two of these faces intersect, they lie, without loss of generality, in the upper half-space. Thus the entire surface lies in one half-space of the plane containing $f_{1}$. However, this holds for all faces, and so the resulting surface cannot be homeomorphic to a torus.

We are interested in representing a toroidal map where we keep a form of convexity of the faces (as defined precisely in Section 2),

but relax the condition that the points in a face are all coplanar. The vertices of the map are represented by points in $\mathbb{R}^{3}$, the edges of the map by straight-line segments, and the faces by the convex hull of their boundary vertices. We require that each face has a "viewing direction" given by a vector $\bar{v}$ such that projecting onto the plane orthogonal to $\bar{v}$ maps the boundary of the face bijectively onto the boundary of the convex hull of the projected points, consistent with their orders. In other words, the face does not necessarily have coplanar boundary vertices on the convex hull, but there is a projection where it is represented with coplanar vertices and is convex. This boundary cycle is a "shadow boundary" of the polytope representing the face. We seek to represent the toroidal map by points in space such that individually each face has a shadow boundary. Moreover, we require that for any two distinct faces, the interiors of their convex hulls are disjoint, and that the viewing directions for shadow boundaries are independent. The idea is that the open disk that is the face minus its boundary can be anywhere in the interior of the convex hull of its boundary points. Call such a representation an "exhibition" of the map.

We need one more concept before we give our main result. The face-width of a map, $f w(G)$, is $\min \{|C \cap G|: C$ is a non-contractible cycle in the surface $\}$. A map is polyhedral if it is of face-width at least 3 and the underlying graph $G$ is 3-connected. (Here, the word polyhedral is being used in its topological-graph-theoretic sense, and should not be confused with geometric polyhedra.)

Theorem 1.1. Every polyhedral map on the torus can be exhibited.

A triangular face has three boundary vertices, so all points in their convex hull are coplanar. Hence an exhibition of a triangulation is necessarily a geometric realization (see Proposition 2.2). This answers Grünbaum's conjecture affirmatively for toroidal triangulations:

Corollary 1.2. Every toroidal triangulation has a geometric realization.

This paper is organized as follows. In Section 2 we give our basic definitions and terminology. In Section 3 we give exhibitions of two special maps. These exhibitions not 
only illustrate our ideas, but are key in later proofs. In Section 4 we extend the exhibitions of our two special maps to exhibitions of vertex splittings of these maps. Section 5 shows how to take a shadow boundaried face and extend the exhibition to include a map inside this face. Combining these two sections, Section 6 shows that if a map $G$ contains a surface minor $H$ of maximum degree 4 with each face bounded by a simple cycle, then an exhibition of $H$ can be modified to an exhibition of $G$. Section 7 shows that every toroidal map contains a vertex split of one of our two special maps. We use this and our known exhibitions to prove the main result in Section 8. We digress in Section 9 to discuss how we constructed one of our key ingredients: an exhibition of a map we call the hexahedron. Section 10 gives some conjectures and conclusions.

\section{Definitions and Terminology}

The graphs in this paper are simple and connected. We consider graphs embedded on a surface; as is common, we require our maps to be cellular; that is, $S-G$ is a collection of disjoint open disks. We call such an embedding a map. A map is circular if every face is bounded by a simple cycle. Equivalently, the map is circular if its underlying graph is 2-connected and the embedding is of face-width at least 2. Fixing an orientation of the surface, we can describe any face of a circular map by a cyclic order on its incident vertices.

Recall that an embedding is polyhedral if it is 3-connected and of face-width at least 3 . Polyhedral maps have the property that the intersection of any two closed faces is simply connected. Every polyhedral map is circular.

Let $G$ be a given map. Form $H$ from $G$ by deleting edges and isolated vertices from the graph $G$ while maintaining the property that $H$ is a map. We call $H$ a submap of $G$. If we in addition allow smoothing degree 2 vertices of $G$, that is, replacing a path $u v w$ of length 2 in $G$ with a single edge $u w$ in $H$, then we call $H$ a topological submap of $G$. If we also allow forming a map $H$ by contracting a non-loop edge of a map $G$, we call $H$ a surface minor of $G$.

We want to represent an embedding using vertices in 3-space, with the edges and faces represented by the convex hull of the points representing their incident vertices. We start with some terms about sets of points in $\mathbb{R}^{3}$.

Let $P$ be a set $\left\{x_{1}, \ldots, x_{k}\right\}$ of $k$ points in $n$-space. The convex hull of $P$, denoted $\langle P\rangle$, is the collection of all points of the form $c_{1} x_{1}+c_{2} x_{2}+\cdots+c_{k} x_{k}$ such that $c_{1}+c_{2}+$ $\cdots+c_{k}=1,0 \leq c_{i} \leq 1$. The convex hull is also called a ( $n$-dimensional) polytope. For example, the convex hull of three non-colinear points in $\mathbb{R}^{n}$ is a triangle. Likewise, the convex hull of four non-coplanar points in $\mathbb{R}^{n}$ is a tetrahedron.

A point in the interior of the polytope $\langle P\rangle$ has a presentation of the form $c_{1} x_{1}+c_{2} x_{2}+$ $\cdots+c_{k} x_{k}$ such that $c_{1}+c_{2}+\cdots+c_{k}=1, c_{i}>0$. The interior of a set of non-coplanar points agrees with the interior in the usual topology on $\mathbb{R}^{3}$, and if the points are coplanar but not colinear, it is equivalent to the interior in the usual topology on $\mathbb{R}^{2}$.

Fix a cyclic ordering $C=\left(x_{1}, x_{2}, \ldots x_{k}\right)$ of the points in $P$. We say that $C$ is a shadow boundary of $\langle P\rangle$ if there exists a vector $\bar{v}$ such that when we project the points of $P$ onto the plane perpendicular to $\bar{v}$, the convex hull of the projected points contain each of the points on its boundary in the clockwise order given by $C$. Intuitively, if we look 
at $\langle P\rangle$ from the direction $\bar{v}$, we see the points occur in the clockwise order given by $C$. We call $\bar{v}$ the viewing direction for the shadow boundary. Observe that if $C$ is a shadow boundary of $\langle P\rangle$, then every point of $P$ occurs on the boundary of the polytope $\langle P\rangle$.

Next consider a set of $n$ points in 3 -space, and a collection $P_{1}, P_{2}, \ldots, P_{m}$ of subsets of these points. We say that the polytopes $\left\langle P_{i}\right\rangle$ are (pairwise) non-overlapping if for each $i, j,\left\langle P_{i} \cap P_{j}\right\rangle=\left\langle P_{i}\right\rangle \cap\left\langle P_{j}\right\rangle$. In other words, the collection is non-overlapping if the interiors of the polytopes are pairwise disjoint. We will also say that a collection of shadow boundaries is non-overlapping if the convex hulls of their underlying point-sets are non-overlapping.

We discuss how to represent a map on a surface by points in Euclidean 3-space $\mathbb{R}^{3}$. A point assignment of a map $G$ is an injection from $V(G)$ to $\mathbb{R}^{3}$. When the point assignment is understood, we freely confuse vertices in $G$ with their associated points in $\mathbb{R}^{3}$. A circular map $G \subset S$ is exhibited by a point assignment if each of the following holds:

1. each facial boundary cycle $C_{i}$ gives a cyclic order that is a shadow boundary of the points $P_{i}$,

2. the associated viewing directions $\bar{v}_{i}$ are independent, and

3. the $\left\langle P_{i}\right\rangle$ 's are non-overlapping.

The main question we investigate is:

Problem 2.1. Which circular embeddings can be exhibited?

An important subclass of graph embeddings are triangulations, where each face is bounded by a 3 -cycle. For each face, its three incident vertices lie in a common plane. The convex hull of these three points must lie in the same plane. This gives the following result.

Proposition 2.2. A triangulation of a surface has an exhibition if and only if it has a geometric realization.

\section{Two Special Exhibitions}

In this section we describe exhibitions of two particular toroidal maps-these exhibitions will turn out to be crucial to our main result. We first define a graph called a $(3 \times 3)$-grid. The vertices are the elements of $\mathbb{Z}_{3} \times \mathbb{Z}_{3}$, where $\mathbb{Z}_{3}$ is the cyclic group of order 3; the edges join $(i, j)$ to $(i+1, j)$ and $(i, j)$ to $(i, j+1)$, for $i, j \in \mathbb{Z}_{3}$. This graph has a natural quadrilateral embedding in the torus: it is depicted in Fig. 2 , where a vertex $(i, j)$ is denoted $i j$.

Proposition 3.1. The $(3 \times 3)$-grid can be exhibited.

Proof. Consider the standard model of a torus, where a circle of radius $0<r<R$ and center $(R, 0)$ in the $x y$-plane is rotated around the $y$-axis in 3 -space. We place the points 


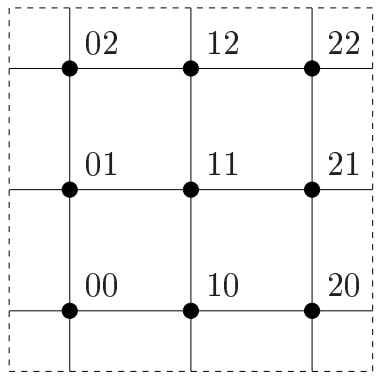

Fig. 2. The $(3 \times 3)$-grid.

equally spaced around this surface; specifically, we use the coordinates

$$
\begin{gathered}
\left(\left[R+r \cos \left(\frac{2 \pi i \theta}{3}\right)\right] \cos \left(\frac{2 \pi j \varphi}{3}\right), r \sin \left(\frac{2 \pi n \theta}{3}\right),\right. \\
\left.\left[R+r \cos \left(\frac{2 \pi n \theta}{3}\right)\right] \sin \left(\frac{2 \pi n \varphi}{3}\right)\right)
\end{gathered}
$$

for the point $(i, j)$, where $i, j=0,1,2$. The four vertices corresponding to a face of a $(3 \times 3)$-grid are coplanar. With the obvious viewing directions for the shadow boundaries of each face, this gives our desired exhibition, as shown in Fig. 3. This is the usual "triangular picture frame".

We now describe our second special complex; we start with the graph of the octahedron. The vertex set is $\{0,1,2,3,4,5\}$, and the only edges not in the graph are $\{i, i+3\}$, where the vertices are read modulo 6 . The octahedron embeds in the torus as shown in Fig. 4. We call this embedding the hexahedron due to that fact that it has six vertices and six faces. Observe that the cyclic permutation $\left(\begin{array}{llll}0 & 1 & 2 & 3\end{array} 45\right)$ acts transitively on this map.

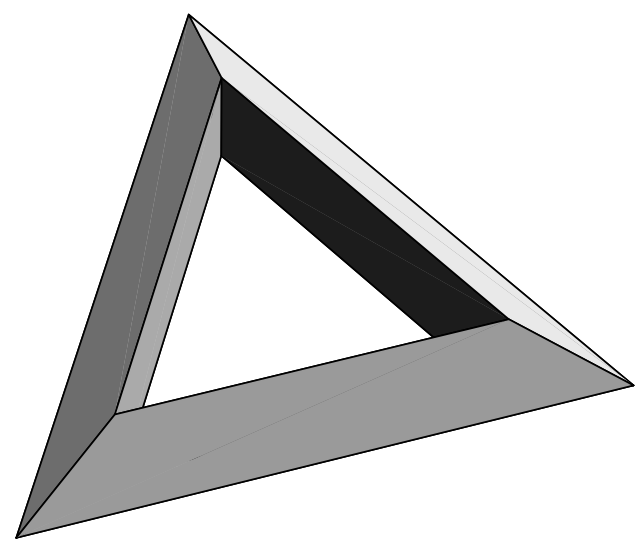

Fig. 3. An exhibition of the $(3 \times 3)$-grid: the triangular picture frame. 


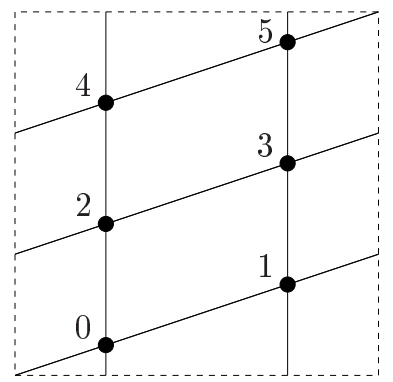

Fig. 4. The octahedron graph embedded on the torus: the hexahedron

Proposition 3.2. The hexahedron can be exhibited.

Proof. The points $0,1,2,3,4,5$ in the graph are mapped respectively to the following points in 3-space:

$$
(0,0,0),(-1,-1,2),(0,3,0),(1,1,0),(3,0,0),(-1,-1,-2) .
$$

It is tedious but routine to verify that this point assignment is the desired exhibition. Figure 5 represents this exhibition. (The solid lines are those of the hexahedron; the role of the dashed lines will be explained in Section 9.) No four points on a face are coplanar, so their convex hull is a tetrahedron. Any tetrahedron with vertices $a, b, c, d$ has a viewing direction with shadow boundary in cyclic order $(a, b, c, d)$ : take the vector (or its negative) whose tail is the midpoint of the line segment $a c$ and whose head is the midpoint of $b d$. Moreover, by varying the head and tail slightly along these line segments from the midpoint, any small pertubation of this viewing direction is also a viewing direction. Hence the viewing directions can be chosen to be pairwise linearly independent.

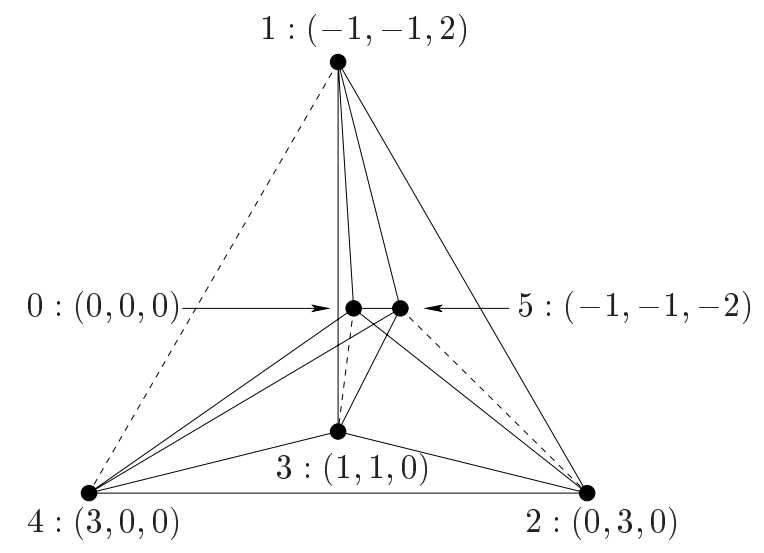

Fig. 5. An exhibition of the hexahedron viewed from the point $(1,2,3)$. 
The proof given, while correct, is unsatisfying. Where did these coordinates come from? We show their derivation in Section 9 which provides an alternate proof that they give an exhibition of the hexahedron. The method in Section 9 combines cyclic polytopes and Schlegel diagrams.

\section{Exhibitions and Edge Contractions}

We now turn our attention to exhibitions of circular maps $G$ and exhibitions of circular maps $H$ formed by contracting a single edge to a point. If $G$ is a graph embedded on a surface $S$ and $e$ is an edge of $G$ that is not a loop, then the edge contraction $H=G / e$ has a natural embedding on $S$. We call this map a surface minor of the original map. We believe the following is true.

Conjecture 4.1. Let $G$ be a circular map and let $H$ be a circular surface minor formed by contracting a single edge of $G$. If $H$ can be exhibited, then $G$ can be exhibited.

The idea is to take an exhibition of $H$ and to modify it slightly at the vertex representing the contracted edge to create an exhibition of $G$. This seems to be difficult to do in general when the degree of the vertex representing the contracted edge is 6 or more, since modifying a single vertex of $H$ does not necessarily give an exhibition of $G$. However, we can prove the conjecture in the following special case.

Theorem 4.2. Let $G$ be a circular map, let $H$ be a circular map formed by contracting a single edge e incident with two degree 3 vertices of $G$. If $H$ can be exhibited, then $G$ can be exhibited.

Proof. We label the relevant vertices, edges, and faces as shown in Fig. 6. Specifically, let $u_{1}$ and $u_{2}$ be the vertices incident with the contracted edge $e$. Label the faces incident with an end of $e$ by $F_{1}, F_{2}, F_{3}, F_{4}$ such that $F_{1}$ is incident with $u_{1}, F_{3}$ with $u_{2}$, and $F_{2}, F_{4}$ are incident with both vertices. Let $e_{i}$ be the edge incident with $F_{i}$ and $F_{i+1}$. The surface
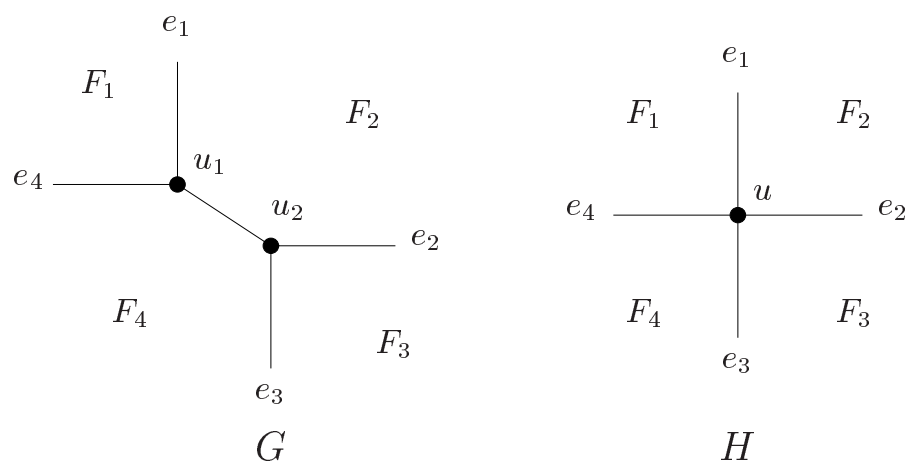

Fig. 6. Contracting an edge to form a surface minor. 
minor $H$ is formed by contracting $u_{1} u_{2}$ to a single vertex $u$, and inherits the labeling of the incident edges and faces, with $u$ denoting the identification of $u_{1}$ and $u_{2}$.

Our goal is to modify an exhibition of $H$ to make an exhibition of $G$. For convenience we call the point in 3-space by the same name as the vertex in the graph.

In the exhibition of $H$, let $\bar{v}_{i}$ denote the viewing direction for the shadow boundary of the face $F_{i}$. We construct the exhibition of $G$ by replacing the point $u$ by two carefully chosen points $u_{1}$ and $u_{2}$ at a small distance from $u$. All other points remain the same, and the vectors $\bar{v}_{i}$ remain the same. Replacing $u$ by $u_{1}$ and $u_{2}$ in this way will ensure that $F_{1}$ and $F_{3}$ have shadow boundaries with viewing direction $\bar{v}_{1}$ and $\bar{v}_{3}$, respectively.

We begin with a sphere $S_{\varepsilon}$ centered at $u$ with radius $\varepsilon$. If $\varepsilon$ is sufficiently small, then any face $F_{i}$ incident with $u$ will still have a shadow boundary with the same viewing direction when $u$ is replaced by an arbitrary $u^{\prime}$ on the surface of $S_{\varepsilon}$. We place both $u_{1}, u_{2}$ on this surface. The care comes in ensuring that the two faces $F_{2}, F_{4}$ incident with both $u_{1}$ and $u_{2}$ have shadow boundaries with viewing directions $\bar{v}_{2}$ and $\bar{v}_{4}$. In many (but not all) situations we will choose $u_{1}$ and $u_{2}$ to be antipodal points on $S_{\varepsilon}$.

Let $s_{i}$ denote the point where $e_{i}$ intersects $S_{\varepsilon}$. Consider the plane $P_{1}$ (respectively $P_{2}$ ) through $u$ defined by $\bar{v}_{2}$ and $e_{1}\left(\bar{v}_{2}\right.$ and $\left.e_{2}\right)$. Clearly, $P_{1}$ and $P_{2}$ meet $S_{\varepsilon}$ in two great circles meeting at antipodal points $N_{2}$ and $S_{2}$. (Note that a $\bar{v}_{2}$ directed line through $u$ meets $S_{\varepsilon}$ at precisely the points $N_{2}$ and $S_{2}$.) One of these great circles contains $s_{1}$ and the other $s_{2}$. The great circles partition $S_{\varepsilon}$ into two pairs of antipodal regions (four quadrants in all). Let $Q_{2}^{+}$denote the quadrant incident with $s_{1}$ but not $s_{2}, Q_{2}^{-}$the "antipodal" quadrant incident with $s_{2}$ but not $s_{1}$, and $R_{2}$ the quadrant incident with neither $s_{1}$ or $s_{2}$. See Fig. 7.

Now $u_{1}$ can be located anywhere inside $Q_{2}^{+}$and $u_{2}$ anywhere inside $Q_{2}^{-}$so that $F_{2}$ in $G$ is viewed in direction $\bar{v}_{2}$. In fact, we could choose $u_{1}$ and $u_{2}$ to be antipodal in this case. Furthermore, both $u_{1}$ and $u_{2}$ can be located inside $R_{2}$ provided that $u_{2}$ and $s_{2}$ lie on the same side of the plane through the origin and $u_{1}$ contains the vector $\bar{v}_{2}$.

We repeat the above to see how $F_{4}$ can be viewed. Let $P_{3}$ (respectively $P_{4}$ ) be the plane through $u$ defined by $\bar{v}_{4}$ and $e_{3}\left(\bar{v}_{4}\right.$ and $\left.e_{4}\right)$. As before, $P_{3}$ and $P_{4}$ intersect the sphere $S_{\varepsilon}$ in two great circles meeting at antipodal points $N_{4}$ and $S_{4}$. These give quadrants $Q_{4}^{+}$ (incident with $s_{4}$ but not $s_{3}$ ), $Q_{4}^{-}$(incident with $s_{3}$ but not $s_{4}$ ) and $R_{4}$ (incident with neither $s_{3}$ or $s_{4}$ ). We note that the antipodal points $N_{2}, S_{2}$ do not coincide with the antipodal points $N_{4}, S_{4}$ since $\bar{v}_{2}$ and $\bar{v}_{4}$ are linearly independent.

As before, $u_{1}$ can be located anywhere inside $Q_{4}^{+}$and $u_{2}$ anywhere inside $Q_{4}^{-}$so that $F_{2}$ in $G$ viewed in direction $\bar{v}_{2}$. Furthermore, both $u_{1}$ and $u_{2}$ can be located inside

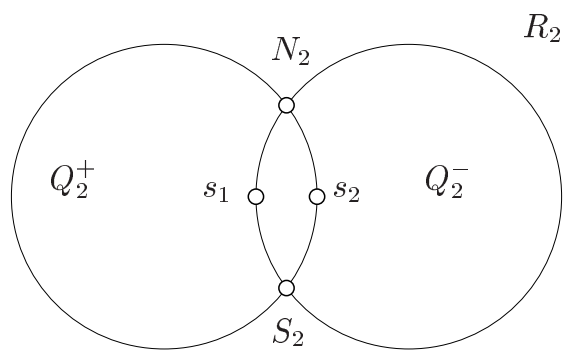

Fig. 7. Great circles on the sphere $S_{\varepsilon}$. 
$R_{4}$ provided that $u_{2}$ and $s_{3}$ lie on the same side of the plane through the origin and $u_{1}$ contains the vector $\bar{v}_{4}$.

We now know where $u_{1}$ and $u_{2}$ can be placed to ensure $F_{2}$ is viewed by $\bar{v}_{2}$ and $F_{4}$ by $\bar{v}_{4}$. If $Q_{2}^{+}$and $Q_{4}^{+}$meet, then we simply choose a point in the interior of their intersection to be the location of $u_{1}$ and $u_{2}$ to be antipodal to $u_{1}$.

We are reduced to the case that $Q_{2}^{+}$and $Q_{4}^{-}$are disjoint. Note that $N_{4}$ and $S_{4}$ are incident with quadrants $Q_{4}^{+}, Q_{4}^{-}$, and $R_{4}$. So if either $N_{4}$ or $S_{4}$ lie in $Q_{2}^{+}$we reduce to the previous case. Hence one of them must lie in $R_{2}$. It follows that $R_{2} \cap R_{4}$ is nonempty. Let $u_{1}$ be any point in the interior of $R_{2} \cap R_{4}$ that is not on the plane through the origin containing $\bar{v}_{2}$ and $\bar{v}_{3}$. It remains to choose a suitable location for $u_{2}$. Consider the plane $P_{2}^{\prime}$ (respectively $\left.P_{4}^{\prime}\right)$ through the origin, $u_{1}$, and containing the vector $\bar{v}_{2}\left(\bar{v}_{4}\right)$. These planes are distinct since $u_{1}$ is not on the plane through the origin with $\bar{v}_{2}$ and $\bar{v}_{3}$. The planes intesect the sphere in two great circles which in turn intersect at $u_{1}$. Now, $s_{2}$ is on one side of $P_{2}^{\prime}$ and $s_{3}$ is on one side of $P_{4}^{\prime}$. We can pick $u_{2}$ anywhere in the intersection of these two sides. By the discussion above, the shadow boundary of $F_{2}$ in $G$ is viewed by $\bar{v}_{2}$ and the shadow boundary of $F_{4}$ in $G$ is viewed by $\bar{v}_{4}$. All viewing directions remain pairwise linear independent, as do any sufficiently small pertubation of the viewing directions.

Finally, we note that the interiors of distinct $\left\langle P_{i}\right\rangle$ are disjoint, which completes the proof.

A split hexahedron is a map embedded on the torus such that we can contract a set of pairwise non-adjacent edges to obtain the hexahedron. Define a split $(3 \times 3)$-grid similarly. The following corollary summarizes the results of this section for use in the rest of the paper.

Corollary 4.3. If $G$ is a split hexahedron or a split $(3 \times 3)$-grid, then $G$ can be exhibited.

\section{Planar Patches in a Polytope}

Consider a topological submap $H$ of a map $G$. Each face $f$ of $H$ contains a portion $G_{f}$ (including the boundary of $f$ ) of the embedded $G$. Given an exhibition of $H$ with a particular face $f$, we want to extend this exhibition to $H \cup G_{f}$. We do this by taking the vertices of $G_{f}$ that are not vertices of $H$ and assigning them points in the convex hull of the points representing the boundary vertices of $f$. In other words, we put $G_{f}$ in the polytope formed by the vertices on the boundary of $f$.

We begin with an examination of the structure of $G_{f}$. Consider a graph $G$ embedded in the plane with a simple cycle $C$ bounding the outside face. Suppose that a subset of the vertices of $C$ are distinguished as major vertices, the remaining ones are minor vertices. We say that the embedded graph is nearly 3-connected if (a) it is 2-connected, (b) the only cut-sets $\{u, v\}$ of size 2 are non-consecutive vertices in $C$, and (c) both components of $G-\{u, v\}$ contain a major vertex. Figure 8 gives an example of such a graph, where the major vertices are the four corners of the bounding polygon. 


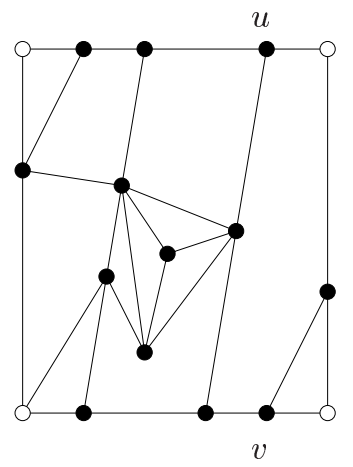

Fig. 8. A nearly 3-connected graph (the non-convex face becomes convex following the application of Proposition 5.2).

Let $G$ be a map and let $H$ be a circular submap. Fix a face $f$ of $H$, let $G_{f}$ be the portion of the embedded $G$ in $f$, and let the major vertices of $G_{f}$ be the vertices of of degree at least 3 in $H$.

Lemma 5.1. Let $G$ be a 3-connected map, and let $H$ be a circular submap. Then $G$ has a circular submap $H^{\prime}$ homeomorphic to $H$ such that for each face $f$ of $H^{\prime}, G_{f}$ is nearly 3-connected.

Proof. Since $G$ is 3-connected and the boundary of $f$ is a cycle, $G_{f}$ is 2-connected. Moreover, the only cut-sets of size 2 are non-consecutative vertices $\{u, v\}$ in $C$. However, it may not be the case that both components of $G_{f}-\{u, v\}$ contain major vertices. If not, call $\{u, v\}$ a bad 2-cut. Pick the submap $H^{\prime}$ of $G$ homeomorphic to $H$ that has the minimum number of bad 2-cuts. We will show that this number is zero.

By way of contradiction, suppose that $H^{\prime}$ has a bad 2-cut. Let $a$ be the major vertex on the boundary of $G_{f}$ closest to $u$, and let $b$ be the major vertex closest to $v$, where we allow the possibilities $a=u$ and $b=v$. Let $[u, v]$ and $[a, b]$ be the parts of the boundary cycle $C$ of $f$ not containing a major vertex. The component of $G-\{u, v\}$ not containing a major vertex is bounded by a simple cycle $C^{\prime}$. Consider the map $H^{\prime \prime}$ formed from $H^{\prime}$ by replacing $[a, b]$ with $([a, b]-[u, v]) \cup\left(C^{\prime}-[u, v]\right)$. In other words, we remove the path joining $u, v$ in $H^{\prime}$ and replace it with the other half of $C^{\prime}$. Note that $H^{\prime \prime}$ is homeomorphic to $H^{\prime}$.

The face in $H^{\prime \prime}$ corresponding to $f$ now has one fewer bad 2-cut. Let $f^{\prime}$ be the other face of $H^{\prime \prime}$ incident with $[u, v]$. Since $G$ is 3 -connected, there must be a path from $[u, v]$ to the other component of $G-\{a, b\}$. Hence $\{u, v\}$ is not a bad 2-cut in $G_{f^{\prime}}$. It follows that $H^{\prime \prime}$ has fewer bad 2-cuts, a contradiction of the minimality of $H^{\prime}$. Hence $H^{\prime}$ has no bad 2-cuts as claimed.

It follows that for each face $f$ of $H^{\prime}, G_{f}$ is nearly 3-connected, and the lemma is shown.

Knowing the structure of $G_{f}$, we examine how to realize this map. 
Proposition 5.2. $\quad$ Let $G_{f}$ be a nearly 3-connected planar map with $k \geq 3$ major vertices and let $C$ be the simple cycle bounding the outside face. Let $D$ be an arbitrary convex $k$-gon in the plane. Then $G_{f}$ can be drawn so that (1) C corresponds to D, (2) the major vertices in $C$ are the corners of $D$, (3) minor vertices in $C$ are evenly spaced between corners, and (4) every face is convex.

Proof. If $G_{f}$ is 3-connected, then the embedding exists by Tutte's theorem [31]. (These "spring embeddings" are found by placing each vertex at the point averaging the coordinates of its neighbors and work for any convex polygonal boundary.) This provides the base case for an induction on the number of cut-sets of order 2. By condition (b) of nearly 3-connected, any 2-cut $\{u, v\}$ is in $C$. Let $G_{1}$ and $G_{2}$ denote the components of $G_{f}-\{u, v\}$ (there are just two since $u$ and $v$ are in $C$ ). We place the vertices of $C$ along $D$ so as to satisfy the first three conditions, and add the edge $u v$ in the interior of $D$. By condition (c) of nearly 3-connected, $u, v$, and the major vertices in $G_{1}$ form a convex polygon. By induction, we convexly embed the subgraph induced by the vertices of $G_{1}$ and $\{u, v\}$ together with the edge $u v$ in this polygon. Here, the vertices $u$ and $v$ play the role of major vertices. We do the same for $G_{2}$. Now, deleting the edge $u v$ if necessary, when it is not in $G$, gives the desired embedding.

We next use this planar representation of $G_{f}$ to find an exhibition of $G_{f}$ in the interior of a polytope that is a shadow boundary of its boundary cycle $C$.

Proposition 5.3. Let $G_{f}$ be a nearly 3-connected planar map with $k \geq 3$ major vertices and let $C$ be the simple cycle bounding the outside face. Suppose that there is a shadow boundary of the vertices of a polytope (or convex polygon) $\langle P\rangle$ in the plane orthogonal to $\bar{v}$ with $C$ as the projected boundary. Then $G_{f}$ has an exhibition such that $C$ is the projected boundary of $\langle P\rangle$, and every face of $G_{f}$ has the same viewing direction $\bar{v}$. Moreover, except for the degenerate case that all of the points of $\langle P\rangle$ are coplanar, the only vertices of $G$ on the boundary of $\langle P\rangle$ are those in $C$, and the only edges on the boundary of $\langle P\rangle$ are those either in $C$ or edges uv on a 2-cut.

Proof. Let $D$ denote the $k$-gon arising from the projection of $C$ in direction $\bar{v}$ onto the plane perpendicular to $\bar{v}$. We begin with the drawing of $G_{f}$ in the planar $k$-gon $D$ given by Proposition 5.2. Let $v$ be a vertex of this planar drawing, and let $\ell_{v}$ be the line (with direction $\bar{v}$ ) through $v$ perpendicular to the plane containing $D$. Now $\ell_{v}$ intersects the boundary of the polytope $P$ in exactly one point if $v$ is in $C$, and in exactly two points otherwise. In the first case we use that point $v^{\prime}$ in our exhibition of $G_{f}$ in $P$. In the second case we set $v^{\prime}$ as the midpoint of the two points of intersection.

If $F$ is the cycle bounding a face in the drawing of $G_{f}$ in $D$, then the polytope determined by the corresponding vertices of $F$ obtained above has a shadow boundary with viewing direction $\bar{v}$. The polyhedra arising in this way from two different faces do not have a point of their interiors in common; if so, then it would project to a point in the interior of two different faces of $G_{f}$ in $D$. Finally, the only vertices on the boundary of $P$ are those corresponding to vertices of $C$, and the only edges on the boundary are those with both ends on $C$. 


\section{Surface Minors of Toroidal Maps}

In this section we examine the relation between exhibitions of a surface minor $H$ of a map $G$ and exhibitions of all of $G$. Basically, we combine the results of Sections 4 and 5 in a general easily applied form. Our goal is the following theorem.

Theorem 6.1. Let $H$ be a surface minor of $G$, where $H$ is a circular map and $G$ is a polyhedral map. Suppose that $H$ has maximum degree at most 4 . If $H$ has an exhibition, then $G$ has an exhibition.

Proof. We can assume that $G$ does not contain any vertices of degree 2. Since $G$ contains $H$ as a minor and $H$ is of maximum degree at most 4, then $G$ contains a submap $H^{\prime}$ together with a collection of pairwise non-adjacent edges $e_{1}, \ldots, e_{k}$ of $H$ such that simultaneously contracting each $e_{i}$ in $H^{\prime}$ gives a map homeomorphic to $H$. We are given that $H$ has an exhibition, so by repeated application of Theorem $4.2 H^{\prime}$ has an exhibition.

Consider a face $f$ of the embedded $H^{\prime}$. Let $P_{f}$ denote the polytope that is the convex hull of the points representing the boundary of $f$. Let $G_{f}$ be that portion of $G$ that is embedded in a face $f$. By Lemma 5.1, $G_{f}$ is nearly 3-connected. By Proposition 5.3, $G_{f}$ can be exhibited in the interior of $P_{f}$, with the only portion of $G_{f}$ on the boundary of $P_{f}$ being either a portion of the cycle bounding $f$, or a chord connecting two non-adjacent vertices $u, v$ on the boundary of $f$. In the latter case $\{u, v\}$ is a cut-set of $G_{f}$.

Consider the point representation of the vertices of $G$ created by Proposition 5.3 applied to each of the faces of $H^{\prime}$. This point assignment satisfies most, but not all, of the requirements for an exhibition of $G$. Suppose that we are given two faces $f_{1}, f_{2}$ of $G$ that lie in different faces of $H^{\prime}$. Then the interiors of the convex hulls corresponding to these faces are disjoint, since the interiors of the corresponding polyhedra are disjoint in the exhibition of $H^{\prime}$. Similarly, if $f_{1}, f_{2}$ lie in the same face of $H^{\prime}$, the interiors are disjoint by Propostion 5.3.

Let $f_{1}^{\prime}, f_{2}^{\prime}$ be two adjacent faces of $H^{\prime}$. They share a common boundary walk $W$. The vertices of $G_{f_{1}^{\prime}}$ and $G_{f_{2}^{\prime}}$ are evenly spaced along $W$, so any two faces of $G$ incident with an edge of $W$ intersect in only that edge.

There cannot be two disjoint faces of $H^{\prime}$ that both contain a common chord $u v$, since $G$ does not contain multiple edges.

The only remaining possibility for two polytopes to intersect improperly is if $H^{\prime}$ has an edge $u v$, and $u v$ also occurs as a cut-set of size 2 in a face not incident with that edge. For example, we refer the reader to Fig. 4, where the edge 23 appears as an edge in the hexahedron $H^{\prime}$, but also might be a chord in the face 2134. Since $G$ contains no parallel edges, the edge $u v$ of $H^{\prime}$ corresponds to a path $W=v_{1} v_{2} \cdots v_{k}$ in $G$, where $u=v_{1}$ and $v=v_{k}$. An edge $v_{i} v_{i+1}$ is incident with two faces $f_{1}, f_{2}$ of $G$, and the edge $u v$ is incident with faces $f_{3}, f_{4}$ of $G$. The convex hulls of say $f_{1}$ and $f_{2}$ both contain the edge $v_{i} v_{i+1}$, which cannot occur in an exhibition.

In this case we perturb the points $v_{2}, \ldots, v_{k-1}$ slightly so that there is no point interior to $W$ that also lies on the chord $u v$, maintaining that the interiors of the convex hulls of different points stay disjoint. We do this by perturbing a point at a time along the path $W$. We assume that each vertex of $W$ is of degree 3 in $G$, since we can replace any vertex of 


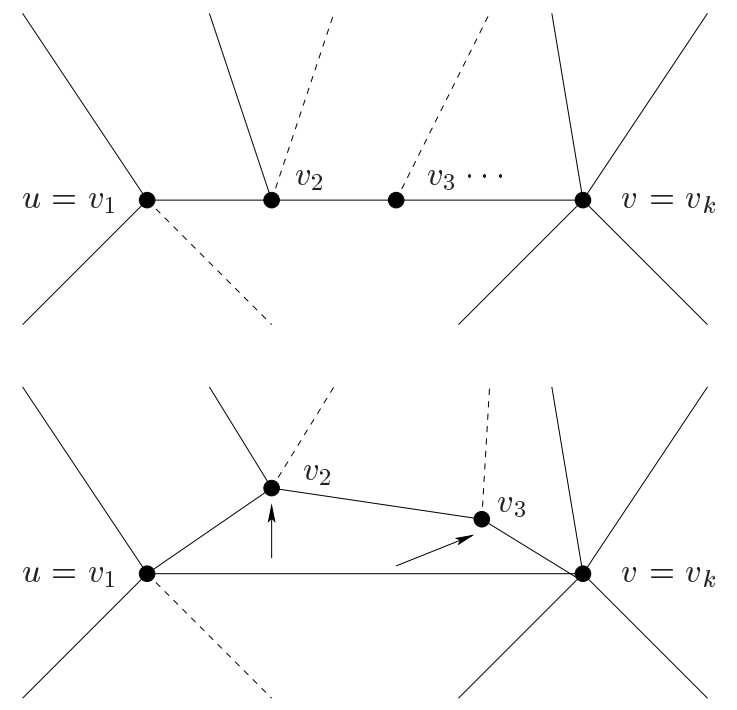

Fig. 9. How to perturb intersections.

degree 2 by an edge joining its two adjacent vertices. We show how to perturb $v_{2}$. Once it is perturbed, we can perturb $v_{3}$, then $v_{4}$, etc.

There are two cases to consider: either $v_{2}$ is of degree 3 or more in both $f_{1}$ and $f_{2}$, or it is of degree 3 or more in (say) $f_{1}$ and degree 2 in $f_{2}$. The first case is easier. There is an $\varepsilon_{1}$ such that perturbing $v_{2}$ by at most $\varepsilon_{1}$ gives a exhibition of $G_{f_{1}}$. Similarly define $\varepsilon_{2}$ for $G_{f_{2}}$. Now perturb $v_{2}$ by some $\varepsilon<\min \left\{\varepsilon_{1}, \varepsilon_{2}\right\}$.

The second case is slightly more subtle. Since $v_{2}$ is of degree 2 in $f_{2}$, we have to perturb $v_{2}$ in a direction that does not make its incident face in $G_{f_{2}}$ non-convex. We perturb as shown in Fig. 9 so that the vertex does not move into the interior of the convex hull of the points representing $f_{1}$. Such a direction always exists.

\section{Finding Submaps of Toroidal Maps}

In earlier sections we have given exhibitions of the split hexahedron and the split $(3 \times 3)$-grids. We showed how such exhibitions can be extended from submaps $H$ to the entire map $G$. In this section we show that any polyhedral map on the torus contains one of our special submaps (note that there are circular maps that do not contain one of our submaps, for example a subdivided $(2 \times 2)$-grid). To illustrate the idea, the Heawood map in Fig. 1 contains the split hexahedron submap. To see this, delete the dashed edge in this figure, suppressing the resulting degree 2 vertices, and contract the heavy edges.

We first need a preliminary lemma. It says, in essence, that if a graph on the torus contains three disjoint non-contractible cycles in two different directions, then it has a $(3 \times 3)$-grid minor.

Lemma 7.1. Let $G$ be a graph embedded on the torus. Suppose that $G$ contains three pairwise disjoint cycles $C_{1}, C_{2}, C_{3}$ and three pairwise disjoint cycles $C_{1}^{\prime}, C_{2}^{\prime}, C_{3}^{\prime}$. Suppose 
that there is a cycle $C$ homotopic to $C_{1}$ and $C^{\prime}$ homotopic to $C_{1}^{\prime}$ with $\left|C \cap C^{\prime}\right|=1$. Then $G$ has a split $(3 \times 3)$-grid as a topological submap.

Proof. It suffices to show that $C_{i} \cap C_{j}^{\prime}$ is simply connected for each $i, j$. Pick the submap $H$ of $G$ with the total minimum number of components in $C_{i} \cap C_{j}^{\prime}$. If $C_{i} \cap C_{j}^{\prime}$ is not simply connected for some $i, j$, then there is a path $P_{i}$ in $C_{i}$ and $P_{j}^{\prime}$ in $C_{j}^{\prime}$ such that $P_{i} \cup P_{j}^{\prime}$ is contractible. Choose $P_{i}$ and $P_{j}^{\prime}$ to enclose the minimum number of faces of the map $G$. Replacing $P_{j}^{\prime}$ with $P_{i}$ in $C_{j}^{\prime}$ gives a submap that contradicts the minimality of $H$.

We can now present the main result of this section.

Theorem 7.2. Every polyhedral toroidal map contains either a split hexahedron or a split $(3 \times 3)$-grid.

Proof. Let $G$ be a polyhedral toroidal map. By Schrijver's theorem [28] any map on the torus of face-width $r$ contains at least $\lfloor 3 r / 4\rfloor$ pairwise disjoint non-contractible cycles; these cycles are necessarily homotopic. Here $r \geq 3$, so $G$ contains two pairwise disjoint non-contractible cycles. Call these cycles $C_{1}$ and $C_{2}$.

We next describe how to cut an embedding along a non-contractible cycle $C$. Begin with a non-contractible cycle $C=\left(v_{1}, \ldots, v_{k}\right)$ of minimal length; such a cycle can have no chords. A small neighborhood $C$ is homeomorphic to a cylinder; $\operatorname{direct} C$ so it has a left side and a right side. Replace each $v_{i}$ by two vertices $v_{i}^{\prime}, v_{i}^{\prime \prime}$, replace each edge $u v_{i}$ on the left side by $u v_{i}^{\prime}$, each $u v_{i}$ on the right by $u v_{i}^{\prime \prime}$, and add in two cycles $C^{\prime}=\left(v_{1}^{\prime}, \ldots, v_{k}^{\prime}\right)$ and $C^{\prime \prime}=\left(v_{1}^{\prime \prime}, \ldots, v_{k}^{\prime \prime}\right)$.

We cut along the cycle $C_{1}$. The result is a planar graph with two distinguished faces, one corresponding to the left side of $C_{1}$ and the other to the right side. We picture it so that the first of these faces contains the origin of the plane, and the second is the outside face. Place a new vertex 0 in the interior of the first face and add edges from 0 to all vertice on the face's boundary cycle. Similarly add a new vertex $\infty$ in the outside face together with edges from $\infty$ to every vertex on the outside face's boundary. Call the resulting graph $\bar{G}$ as shown in Fig. 10.

It is not hard to show that $\bar{G}$ is 3-connected. In particular, there exist three internally disjoint paths from 0 to $\infty$ by Menger's theorem [23]. Let $a^{\prime}, b^{\prime}, c^{\prime}$ denote the last vertex on these paths on the left side of $C_{1}$, and $x^{\prime \prime}, y^{\prime \prime}, z^{\prime \prime}$ the first vertex (respectively) on these paths on the right side of $C_{1}$. Let $a, b, c, x, y, z$ respectively denote the vertices in $C_{1}$ corresponding to $a^{\prime}, b^{\prime}, c^{\prime}, x^{\prime \prime}, y^{\prime \prime}, z^{\prime \prime}$. In particular, there are pairwise disjoint $a x-, b y-$, and $c z$-paths in $G$.

We examine the cyclic order of $a, b, c, x, y, z$ along $C_{1}$. For convenience, consider the directed hexagon formed from $C_{1}$ by smoothing all other vertices and directing by the cyclic order induced by $(a, b, c)$. We allow the possibility that some of $\{a, b, c\}$ are equal to some of $\{x, y, z\}$. Without loss of generality, there are three cases to consider (see Fig. 11):

Case 1: The hexagon contains arcs $x b, y c, z a$, including the possibilities $x=b, y=c$, and/or $z=a$. In this case we get a split hexahedron and the theorem is satisfied. 


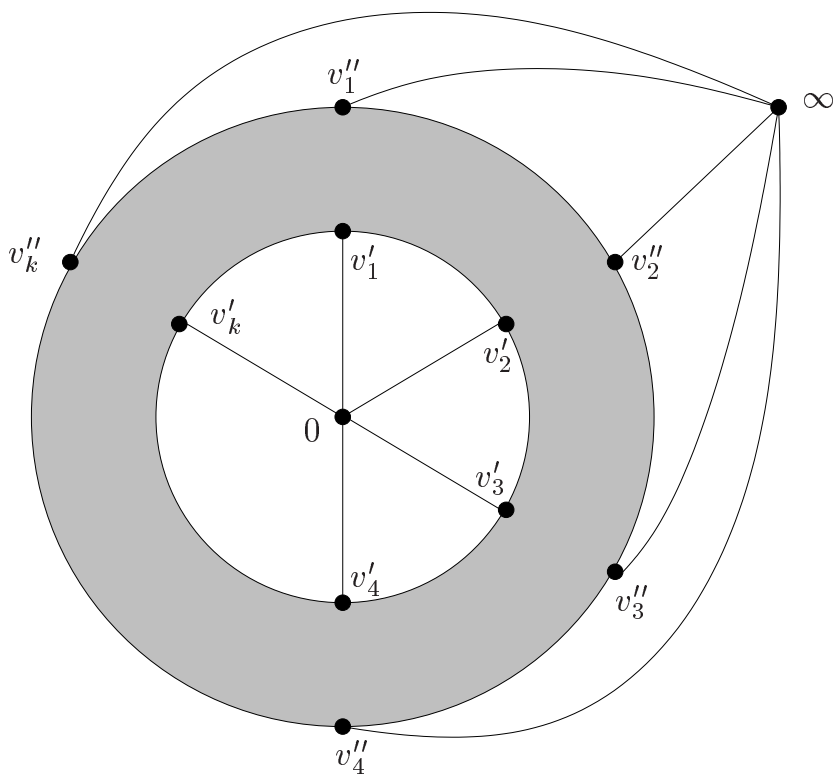

Fig. 10. Cutting along a non-contractible cycle.

Case 2: The hexagon contains arcs $a x, y b, z c$ and similar subcases. In this case $G$ contains three non-contractible pairwise disjoint cycles. We examine this case later.

Case 3: The vertices appear in cyclic order $(a, b, c, x, y, z)$, where possibly $c=x$ and $z=a$. In this case we begin again: Let $a_{2}, b_{2}, c_{2}$ denote the last vertex on the $a x-, b y-$, and $c z$-paths on $C_{2}$ (see Fig. 11(c)), and $x_{2}, y_{2}, z_{2}$ the first vertex (respectively) on $C_{2}$. This time we cut along cycle $C_{2}$ and connect 0 only to the vertices $a_{2}^{\prime}, b_{2}^{\prime}, c_{2}^{\prime}$ corresponding to $a_{2}, b_{2}, c_{2}$ on the left of $C_{2}$ and $\infty$ only to vertices $x_{2}^{\prime \prime}, y_{2}^{\prime \prime}, z_{2}^{\prime \prime}$ corresponding to $x_{2}, y_{2}, z_{2}$ on the right of $C_{2}$ (producing $\bar{G}$ ).

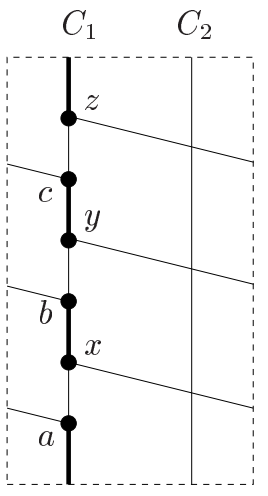

(a) Case 1 .

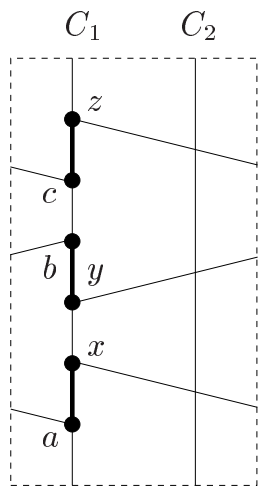

(b) Case 2.

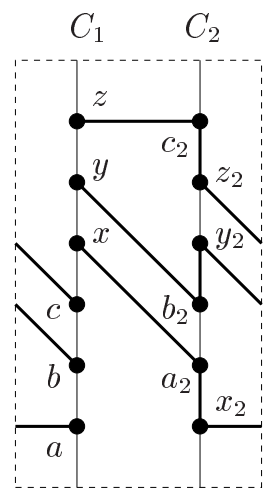

(c) Case 3 .

Fig. 11. The three possibilities for the $a x$-, $b y$-, and $c z$-paths in $G$. 
We will show that there exist three edge-disjoint paths from 0 to $\infty$. Suppose instead that there is a cut-set $\{u, v\}$ of size 2 separating 0 and $\infty$ in $\bar{G}$, and let $G_{1}$ and $G_{2}$ be the components of this cut containing 0 and $\infty$ respectively. Recall that there are three pairwise vertex-disjoint paths from 0 to $C_{1}$, so $C_{1}$ is not contained entirely in $G_{2}$. Likewise, there are three pairwise vertex-disjoint paths from $\infty$ to $C_{1}$, so $C_{1}$ is not contained entirely in $G_{2}$. It follows that $u, v$ are on $C_{1}$. No face incident with $C_{1}$ is also incident with either 0 or $\infty$. Hence the two faces incident with both $u, v$ in $\bar{G}$ are not incident with 0 or $\infty$. In particular, they are faces of the original toroidal embedding of $G$. This contradicts either the fact that $G$ is 3-connected or contradicts that the face-width is at least 3.

We conclude that there are three vertex-disjoint paths from 0 to $\infty$. These three paths intersect $C_{1}$ so as to fall in either Case 1 or Case 2.

We now return to Case 2: $G$ contains three non-contractible pairwise disjoint cycles. Call these $C_{1}, C_{2}$, and $C_{3}$. Again we cut along $C_{1}$. Applying the same logic as before, we either get a split hexahedron, or we get three non-contractible cycles in a different homotopy class. In the latter case Lemma 7.1 applies and we get a split $(3 \times 3)$-grid as desired.

\section{Proof of the Main Result}

We combine the results of the previous sections to prove the main result, given in Section 1.

Theorem 1.1. Every polyhedral map on the torus can be exhibited.

Proof. Theorem 7.2 shows that any polyhedral $G$ has a submap $H$ that is either a split hexahedron or a split $(3 \times 3)$-grid. Corollary 4.3 shows that each such $H$ has an exhibition. By Theorem 6.1 these exhibitions of $H$ extend to exhibitions of $G$. Our main result follows.

\section{Exhibiting a Hexahedron}

In the proof of Proposition 3.2 we described an exhibition of the hexahedron. The description gave the specific points in 3-space, but left lacking the idea behind how we found this exhibition. Discovering this exhibition involved an interesting detour to fourdimensional space $\mathbb{R}^{4}$, which we describe here. We are heavily indebted to Duke [12] and Altshuler [1], who pioneered the method combining cyclic polytopes and Schlegel diagrams. A nice exposition on cyclic polytopes is given in Chapter 4.7 of [17].

We begin with the curve of moments, which is the locus of points $\left(t, t^{2}, \ldots, t^{d}\right)$ in $\mathbb{R}^{d}$. Consider any $d$ distinct non-zero numbers $t_{1}, \ldots, t_{d}$. Let $M$ be the $(d \times d)$-matrix whose $i$ th row is given by the point coordinates $\left(t_{i}, t_{i}^{2}, \ldots, t_{i}^{d}\right), i=1, \ldots, d$. By Vandemonde's theorem, the determinant of this matrix is equal to $\left(t_{1} t_{2} \cdots t_{d}\right) \Pi_{i>j}\left(t_{i}-t_{j}\right)$, and so is non-zero. This in turn implies that the row vectors are linearly independent, and so the convex hull of the $d$ points $\left(t_{i}, t_{i}^{2}, \ldots, t_{i}^{d}\right)$ is a $(d-1)$-simplex. 
Consider any set of $v$ distinct non-zero points on the curve of moments in $\mathbb{R}^{d}$. The convex hull of these points is a simplicial $d$-polytope, called the cyclic polytope $C(v, d)$ by Grünbaum. He relates the following result [13] (see also Section 4.8 of [17] and Section 13.1.4 of [19]) which identifies the facets of this cyclic polytope.

Lemma 9.1 (Gale's Evenness Condition). A simplex $S$ is a facet of $C$ if and only if for every $i, j$, the $i$ th and $j$ th coordinates of $C$ are separated by an even number of points $t_{k}$.

We are interested in the case $v=6$ and $d=4$. We choose the six points $v_{1}, \ldots, v_{6}$ on the curve of moments generated by $t=-3,-2,-1,1,2,3$, respectively. These correspond to the the vertices $0,1,2,3,4,5$ of our hexahedron as labeled in Fig. 4. The points corresponding to the six faces

$$
\{(0,1,3,2),(1,2,4,3),(2,3,5,4),(3,4,0,5),(4,5,1,0),(5,0,4,3)\}
$$

generate six 4-simplices. Each of these satisify Gale's evenness condition, so they are facets of the cyclic polytope. It follows that they form a geometric realization of the hexahedron in 4-space $\left(\mathbb{R}^{4}\right)$.

We now project from 4-space back to 3 -space. We do this with a Schlegel diagram [27] (see also Section 3.3 of [17] and Section 13.1.6 of [19]), a type of stereographic projection. Let $F_{0}$ be a facet of a $d$-polytope $P$ in $\mathbb{R}^{d}$. Pick a point $x_{0}$ just slightly outside of $P$ near the facet $F$. More precisely, chose $x_{0}$ such that the only affine hull of a facet of $P$ that separates $x_{0}$ and $P$ is that of the facet $F_{0}$. Project $P$ onto the affine space generated by $F_{0}$ by rays rooted at $x_{0}$. The result is called the Schlegel diagram of $P$ based at $F_{0}$. For example, Fig. 12 shows a Schlegel diagram of a standard cube in 3-space from any of its faces.

The authors chose the simplex $\{1,2,4,5\}$ for the role of $F_{0}$. This satisfies Gale's Evenness Condition, so it is a facet of the cyclic polytope. It is important not to choose a face of the hexahedron, because the selected facet corresponds to the unbounded region of the Schlegel diagram. We calculated the coordinates of this projection using Mathematica. These coordinates were all rational valued. Scaling by the least common multiple of their denominators gave us integer coordinates for realizing the hexahedron. These coordinates were then modified by Grünbaum (personal communication) to give the smaller magnitude integer coordinates of Proposition 3.2.

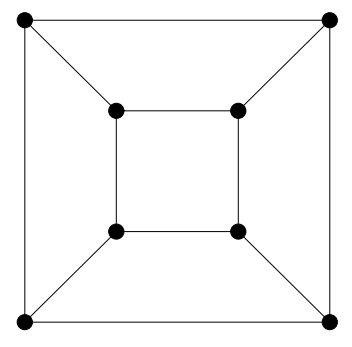

Fig. 12. A Schlegel diagram of a cube. 


\section{Conjectures and Conclusions}

We begin with a series of comments about toroidal triangulations. If one is interested only in toroidal triangulations, instead of arbitrary polyhedral maps, the following proposition is helpful. It shows that we do not need to consider embeddings of a split $(3 \times 3)$-grid.

Proposition 10.1. Every toroidal triangulation contains a split hexahedron.

Proof. Lavrenchenko [20] found the 21 toroidal triangulations that are minimal under the minor order; this was reportedly done in unpublished work by Grünbaum and Duke (see [16]). It is straightforward to check that each of these 21 maps has a hexahedron. If $H$ is a surface minor of $G$ and $H$ has a hexahedron, then $G$ has a split hexahedron.

We offer the following stronger conjecture.

Conjecture 10.2. Every toroidal triangulation contains a hexahedron.

It is tempting to conjecture an even stronger result: given two homotopic disjoint non-contractible cycles in a toroidal triangulation, there exists a hexahedron that contains both cycles. However, this stronger statement is false, as shown in Fig. 13. The graph is $K_{2,2,2,2}$. The two disjoint non-contractible cycles are the horizontal 4-cycles.

We note that not every toroidal triangulation contains a split $(3 \times 3)$-grid. The toroidal embedding of the complete graph $K_{7}$ is an example.

The unique triangulation of the torus by $K_{7}$ can be realized by gluing together seven tetrahedra along some of their faces. This motivates the following conjecture.

Conjecture 10.3. Every triangulation of the torus can be realized geometrically by fitting together tetrahedra such that each vertex of a tetrahedron is a vertex of the triangulation.

Altshuler and Brehm [2] showed that there is a toroidal map that is not a Schlegel diagram of a 4-polytope. Hence the techniques of this paper may not be enough to prove this conjecture, since these techniques rely on an exhibition of the hexahedron as a Schlegel diagram.

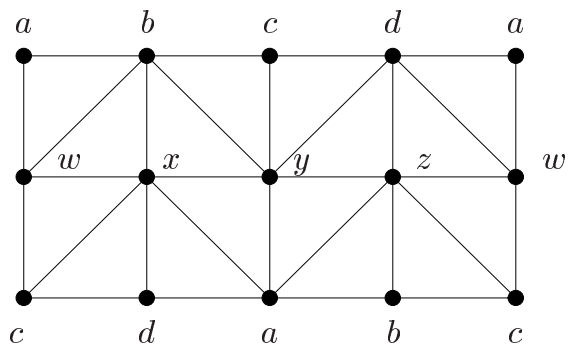

Fig. 13. An interesting toroidal triangulation. 
What about surfaces other than the torus? Recall that Bokowski and Guedes de Oliveira [6] showed that there is a triangulation of the surface of genus 6 that has no geometric realization, and hence has no exhibition. In fact, Bokowski reports (personal communication) that no triangulation of the surface of genus 6 by $K_{9}$ has a geometric realization. It is an easy modification of one example to find a trianguation of genus $g$ for each $g \geq 6$ that has no geometric realization. The existence of such examples is open for $g$ between 2 and 5 . We ask about the simplest open genus.

Question 10.4. Does every triangulation of the double torus have a geometric realization?

We turn our attention to non-triangulations. Note that for these maps on the torus we needed both the embeddings of the split hexahedron and of the split $(3 \times 3)$-grid. In particular, the hexahedron does not contain a split $(3 \times 3)$-grid, and a $(3 \times 3)$-grid does not contain a split hexahedron.

The situation is different for maps of large face-width.

Theorem 10.5. There is a number $f(g)$ such that every 3-connected map on the surface of genus $g$ having face-width at least $f(g)$ has a geometric realization, in particular, it has an exhibition.

Proof. Nakamoto notes (personal communication) that for each fixed surface, there is a cubic map $H$ that has a geometric realization. By a result of Robertson and Seymour [26] every map $G$ of sufficiently large face-width contains $H$ as a surface minor. Since $H$ is cubic, the map $G$ must also contain $H$ as a topological minor. It follows that, by our methods, $G$ has a geometric realization, which is also an exhibition.

The preceding theorem is especially interesting for triangulations. Having shown that such a function $f(g)$ exists for general polyhedral maps, it is natural to ask the following.

Question 10.6. What is the asymptotic behavior of $f(g)$ ? Could it be constant?

We believe the answer to the second question is no.

Finally, we consider the non-orientable surfaces. Triangulations of these surfaces do not have geometric realizations, since they do not embed in $\mathbb{R}^{3}$. However, suppose that we remove the interior of one face from a triangulation of the projective plane. The result is a triangulation of the Möbius band. Not every triangulation of the Möbius band has a geometric realization in $\mathbb{R}^{3}$, more strongly, they may not have geometric immersions (allowing self-intersections) [10].

Question 10.7. Which triangulations of the Möbius band have a geometric realization?

Bonnington et al. [7] have shown that any triangulation of the projective plane has a face $f$ such that the triangulation of the Möbius band created by deleting the interior of $f$ has a geometric realization.

For other unsolved problems along these lines see [25]. 


\section{Acknowledgements}

We thank Serge Lavrenchenko for introducing us to this problem and for helpful discussions. We are deeply indebted to both the second referee and Ulrich Brehm who independently identified a gap in an earlier proof of Theorem 4.2. This resulted in us making an important modification to the definition of exhibition. Finally, we sincerely thank Jürgen Bokowski, Branko Grünbaum, Jörg Wills, and Günter Ziegler for many

helpful comments on the manuscript, and Branko Grünbaum for the inclusion of his Fig. 5.

\section{References}

1. A. Altshuler, Polyhedral realization in $\mathbb{R}^{3}$ of triangulations of the torus and 2-manifolds in cyclic 4polytopes, Discrete Math. 1 (1971), 211-238.

2. A. Altshuler and U. Brehm, A non-Schlegelian map on the torus, Mathematika 31(1) (1984), 83-88.

3. D. W. Barnette, Coloring polyhedral manifolds, in Discrete Geometry and Convexity (New York, 1982), pp. 192-195, Annals of the New York Academy of Sciences, vol. 440, New York Academy of Sciences, New York, 1985.

4. U. Betke and P. Gritzmann, A combinatorial condition for the existence of polyhedral 2-manifolds, Israel J. Math. 42(4) (1982), 297-299.

5. J. Bokowski and A. Eggert, Toutes les réalisations du tore de Möbius avec sept sommets, Structural Topology, No. 17 (1991), 59-78.

6. J. Bokowski and A. Guedes de Oliveira, On the generation of oriented matroids, Discrete Comput. Geom. 24 (2000), 197-208.

7. C.P. Bonnington, A. Nakamoto, and K. Ohba, Geometric realizations of a triangulation on the projective plane with one face removed, preprint.

8. U. Brehm and G. Schild, Realizability of the torus and the projective plane in $\mathbb{R}^{4}$, Israel J. Math. 91 (1995), 249-251.

9. U. Brehm and E. Schulte, Polyhedral maps, in Handbook of Discrete and Computational Geometry, pp. 345-358 (eds. J.E. Goodman and J. O'Rourke), CRC Press, Boca Raton, FL, 1977.

10. U. Brehm and J.M. Wills, Polyhedral manifolds, in Handbook of Convex Geometry, pp. 535-554 (eds. P.M. Gruber and J.M. Wills), North Holland, Amsterdam, 1993.

11. Á. Császár, A polyhedron without diagonals, Acta. Sci. Math. (Szeged) 13 (1949), 140-142.

12. R.A. Duke, Geometric embeddings of complexes, Amer. Math. Monthly 77 (1970), 597-603.

13. D. Gale, Neighborly and cyclic polytopes, pp. 225-232, in Proceedings of Symposium in Pure Mathematics, vol. 7 (Convexity), American Mathematical Society, Providence, RI, 1963.

14. M. Gardner, On the remarkable Császár polyhedron and its applications, Scientific American (March 1975), 102-107.

15. M. Gardner, Modern minimal art, Scientific American (November 1978), 20-24.

16. B. Grünbaum, Acoptic polyhedra, in Advances in Discrete and Computational Geometry, pp. 163-199 (eds. B. Chazelle, J.E. Goodman, and R. Pollack), Contemporary Mathematics, vol. 223, American Mathematical Society, Providence, RI, 1999.

17. B. Grünbaum, Convex Polytopes, second edition, Graduate Texts in Mathematics, vol. 221, Springer, New York, 2003.

18. B. Grünbaum and G.C. Shephard, Duality of polyhedra, in Shaping Space: a Polyhedral Approach, pp. 205-211 (eds. M. Senechal and G. Fleck), Birkhäuser, Boston, MA, 1988.

19. M. Henk, J. Richter-Gebert, and G. Ziegler, Basic properties of convex polytopes, in Handbook of Discrete and Computational Geometry, pp. 243-270 (eds. J.E. Goodman and J. O'Rourke), CRC Press, Boca Raton, FL, 1977.

20. S. Lavrenchenko, Irreducible triangulations of a torus (Russian), Ukrain. Geom. Sb. 30 (1987), 52-62. Translation in J. Soviet Math. 51(5) (1990), 2537-2543.

21. P. McMullen, Ch. Schulz and J.M. Wills, Equivelar polyhedral manifolds in E3, Israel J. Math. 41(4) (1982), 331-346. 
22. P. McMullen, Ch. Schulz, and J.M. Wills, Polyhedral 2-manifolds in E3 with unusually large genus, Israel J. Math. 46(1-2) (1983), 127-144.

23. K. Menger, Zur allgemeinen Kurventheorie, Fund. Math. 10 (1927), 95-115.

24. A.F. Möbius, Über die bestimmung des inhalts eines polyeders, Berichte K. Sachs. Ges. Wiss., Math. Phys. Klass 17 (1865), 31-68.

25. J. Reay, Can neighborly polyhedra be realized geometrically?, in Shaping Space: a Polyhedral Approach, pp. 251-253 (eds. M. Senechal and G. Fleck), Birkhäuser, Boston, MA, 1988.

26. N. Robertson and P. D. Seymour, Graph minors. VII. Disjoint paths on a surface, J. Combin. Theory Ser. B 45(2) (1988), 212-254.

27. V. Schlegel, Theorie der homogen zusammengesetzten Raumgebilde, Nova Acta Leop. Carol. 44 (1883), 343-459.

28. A. Schrijver, Graphs on the torus and the geometry of numbers, J. Combin. Theory Ser. B $\mathbf{5 8}$ (1993), $147-158$

29. E. Steinitz, Polyeder un Raumeinteilunger, Enzykl. Math. Wiss. Vol. 3, Teil 3A612 (1922), 1-139.

30. L. Szilassi, Regular toroids, Structural Topology 12 (1986), 69-80.

31. W. T. Tutte, How to draw a graph, Proc. London Math. Soc. (3), 13 (1963), 743-767.

32. E. W. Weisstein, Császár Polyhedron, From MathWorld-A Wolfram Web Resource, http://mathworld.wolfram.com/CsaszarPolyhedron.html.

33. E. W. Weisstein, Szilassi Polyhedron, From MathWorld-A Wolfram Web Resource, http://mathworld.wolfram.com/SzilassiPolyhedron.html.

Received July 19, 2004, and in revised form March 22, 2007. Online publication August 27, 2007. 